EXTENDED REPORT

\title{
A case control study of age related macular degeneration and use of statins
}

\author{
L Smeeth, C Cook, U Chakravarthy, R Hubbard, A E Fletcher
}

Br J Ophthalmol 2005;89:1171-1175. doi: 10.1136/bjo.2004.064477

See end of article for authors' affiliations .....................

Correspondence to: Dr L Smeeth, Department of Epidemiology and Population Health, London School of Hygiene and Tropical Medicine, Keppel Street, London WC'1E 7HT, UK; liam.smeeth@Ishtm. ac.uk

Accepted for publication 8 April 2005

\begin{abstract}
Aims: Age related macular degeneration (AMD) is the leading cause of blindness in industrialised countries. Previous studies have suggested that statins may have a protective effect against the disease; however, existing studies have had limited power to reliably detect or exclude an effect and have produced conflicting results. The authors assessed the risk of AMD associated with the use of statins.

Methods: Population based case control study using the United Kingdom General Practice Research Database. 18007 people with diagnosed AMD were compared with 86169 controls matched on age, sex, and general practice. The primary outcome was the odds ratio for the association between exposure to statins and AMD.

Results: The crude odds ratio for the association between any recorded exposure to statins and AMD was $1.32(95 \% \mathrm{Cl} 1.17$ to 1.48 ), but this reduced to 0.93 (95\% Cl 0.81 to $1.07, \mathrm{p}=0.33$ ) after adjustment for consultation rate, smoking, alcohol intake, body mass index, atherosclerotic disease, hyperlipidaemia, heart failure, diabetes mellitus, hypertension, use of other cardiovascular drugs, and use of fibrates. There was no evidence that the risk varied by dose of statin, duration of use, or that the risk varied for individual statins.

Conclusion: In the short and medium term statin use is not associated with a decreased risk of AMD. Whether subgroups of patients with specific forms of AMD (particularly choroidal neovascularisation) benefit from statin therapy remains a possibility.
\end{abstract}

A ge related macular degeneration (AMD) is the leading cause of blindness in industrialised countries. ${ }^{1}$ The ageing of the population in developing countries will increase the importance of AMD in poorer areas of the world. The early manifestations of AMD which occur in the macular retina are the presence of altered pigmentation and/or yellowish deposits known as drusen. These early clinical signs are usually not accompanied by overt vision loss. However a proportion of people with drusen and pigmentary changes will progress to a state of advanced AMD with the development of choroidal neovascularisation (CNV) and/or geographic atrophy, both of which can have devastating effects on central visual function.

The Age-Related Eye Diseases Study was a large randomised factorial trial which showed that long term oral supplementation with a high dose multivitamin supplement and/or zinc significantly reduced progression to advanced AMD in subjects in the highest risk categories $(23 \%$ intervention $v 28 \%$ placebo). ${ }^{2}$ However some $75 \%$ of people did not benefit. Furthermore, existing treatments for CNV are restricted to a small proportion of affected people as therapies are not uniformly suitable and benefits are modest. ${ }^{3}$ There are currently no treatments for established geographic atrophy. Therefore the majority of subjects at risk will progress to advanced AMD and most of these will experience incapacitating vision loss as the disease is most often bilateral

The aetiology of AMD is obscure and the pathogenic pathways mediating the development of CNV and geographic atrophy are not understood. AMD and atherosclerosis have been shown to share a number of risk factors, ${ }^{4}$ leading to suggestions that they may have similar aetiologies. ${ }^{5}$ In recent years several reports have suggested that statins which are known to be beneficial in vascular disease also exert protective effects in AMD. Notably a small cross sectional survey observed a marked protective effect with an odds ratio of $0.11,95 \%$ CI 0.02 to $0.83 .{ }^{6}$ Another small study found that use of cholesterol lowering drugs was associated with an almost fourfold decrease in the risk of drusen progression, however the result was not significant. ${ }^{7}$ Subsequent studies have produced inconsistent results ${ }^{8-13}$ Some of the inconsistencies in these reports may relate to the lack of adequate power of the smaller studies to reliably estimate the effect of statins on AMD. ${ }^{14}$ The United Kingdom General Practice Research Database contains the prescribing information for several million patients receiving treatment from their general practitioners. We therefore exploited the availability of this database to undertake a matched case control study to assess the risk of AMD associated with exposure to statins.

\section{METHODS}

\section{The General Practice Research Database}

The General Practice Research Database (GPRD), previously known as the VAMP Research Bank, was set up in $1987 .{ }^{15}$ It contains complete prescribing and diagnostic information for over three million people and represents the largest source of continuous data on illness and prescribing habits in the UK. The practices are broadly representative of all practices in England and Wales in terms of geographical distribution and size and the age and sex distributions of the population included in the GPRD are very similar to the whole UK population. ${ }^{16}$ The data available directly from the database include all drug prescriptions, and a record of every consultation and of every diagnosis. Diagnoses made in specialist clinics and hospitals are communicated to the general practitioner in writing and are recorded in the electronic record. The completeness of recording of such diagnoses has been shown to be high. ${ }^{17-20}$ The data collected are audited regularly and the participating general practices

Abbreviations: $A M D$, age related macular degeneration; $C N V$, choroidal neovascularisation; GPRD, General Practice Research Database. 
subjected to a number of quality checks. The quality of the information in the database has been validated in a number of independent studies and has been found to be high. ${ }^{15}$ The information obtained from the database is entirely anonymous. Ethical approval for the study was obtained from the Scientific and Ethical Advisory Group of the GPRD.

\section{Selection of participants}

The source population was all patients registered with general practices who contributed to the GPRD during the period June 1987 to April 2002. The diagnosis of AMD was made by eye specialists and recorded in the electronic health record by general practitioners. Eligible cases were defined as any person aged 50 years or more who had a first diagnosis of AMD while registered with a practice participating in the GPRD. The majority of such cases would therefore be likely to have AMD sufficiently severe as to materially affect their vision. Less than $1 \%$ of the diagnostic codes entered by contributing general practitioners were specific to neovascular AMD or geographic atrophy. We were therefore not able to distinguish early AMD from advanced AMD or CNV from geographic atrophy. The date of the first diagnosis of AMD we called the index date. The observation period was defined as the period between the start of the patient's GPRD record and the index date. Cases were excluded if their index date was before the start of their GPRD observation period or during the first 6 months of this period. For each case, five controls with no record of AMD anywhere in their medical record were selected. Controls were alive and registered with the GPRD on the index date of their matched case and were matched on age within 5 years, sex, and practice. Controls were excluded if the index date of their matched case was within the first 6 months of the control's GPRD observation period or after the end of their observation period. Controls were also excluded if they had no clinical data entered during their GPRD observation period because of concerns about the completeness of the clinical data recorded.

\section{Data processing and analysis}

Data from the electronic records were extracted and set up as a relational database. Only drug exposure and diagnoses made before the index date were included in the analyses. Statin exposure was defined as ever or never based on whether a person had a prescription for any statin drug recorded in their electronic medical record. The mean daily dose of statin was categorised as low (10 mg or less per day), moderate $(20 \mathrm{mg})$, or high (30 $\mathrm{mg}$ or more per day). In addition the total number of prescriptions for any statin was extracted as was information about the type of statin prescribed.

Table 1 Description of cases and controls: univariate associations and adjustment for consultation rate

\begin{tabular}{|c|c|c|c|c|c|c|}
\hline \multirow[b]{2}{*}{ Number (\%) female* } & \multicolumn{2}{|c|}{ Cases $(n=18007)$} & \multicolumn{2}{|c|}{ Controls $(n=86169)$} & \multirow[t]{3}{*}{$\begin{array}{l}\text { Univariate odds } \\
\text { ratio }(95 \% \mathrm{Cl})\end{array}$} & \multirow[t]{2}{*}{$\begin{array}{l}\text { Odds ratio adjusted for } \\
\text { consultation rate }(95 \% \mathrm{Cl})\end{array}$} \\
\hline & 11872 & $(65.9 \%)$ & 56848 & $(66.0 \%)$ & & \\
\hline \multicolumn{6}{|l|}{ Age at index date (years) ${ }^{*}$} & \\
\hline $50-59$ & 718 & $(4.0 \%)$ & 3472 & $(4.0 \%)$ & & \\
\hline $60-69$ & 2379 & (13.2\%) & 11482 & (13.3\%) & & \\
\hline 70-79 & 6862 & $(38.1 \%)$ & 33125 & $(38.4 \%)$ & & \\
\hline $80-89$ & 6897 & $(38.3 \%)$ & 33088 & $(38.4 \%)$ & & \\
\hline $90+$ & 1151 & $(6.4 \%)$ & 5002 & $(5.8 \%)$ & & \\
\hline Annual consultation rate (median (IQR)) & 6.3 & $(3.7-10.1)$ & 4.8 & $(2.3-8.3)$ & & \\
\hline Observation period in years (median (IQR)) & 4.0 & $(2.1-6.5)$ & 3.9 & $(2.1-6.4)$ & & \\
\hline \multicolumn{7}{|l|}{ Smoking status } \\
\hline Non-smoker & 8763 & $(48.7 \%)$ & 40507 & $(47.0 \%)$ & Baseline & \\
\hline Ex-smoker & 2766 & $(15.4 \%)$ & 11468 & $(13.3 \%)$ & $1.19(1.13-1.26)$ & $1.14(1.08-1.20)$ \\
\hline Current smoker & 3161 & $(17.6 \%)$ & 13358 & $(15.5 \%)$ & $1.14(1.09-1.21)$ & $1.17(1.11-1.22)$ \\
\hline Missing data & 3317 & $(18.4 \%)$ & 20836 & $(24.2 \%)$ & $0.67(0.63-0.70)$ & $0.71(0.68-0.75)$ \\
\hline \multicolumn{7}{|l|}{ Drinking status } \\
\hline Non-drinker & 3316 & $(18.4 \%)$ & 15211 & $(17.7 \%)$ & Baseline & \\
\hline Ex-drinker & 30 & $(0.17 \%)$ & 167 & $(0.19 \%)$ & $0.84(0.57-1.25)$ & $0.75(0.50-1.11)$ \\
\hline Current drinker (amount unknown) & 210 & $(1.2 \%)$ & 914 & $(1.1 \%)$ & $1.08(0.92-1.27)$ & $1.11(0.94-1.30)$ \\
\hline Rare drinker ( $<2$ units per day) & 8342 & $(46.3 \%)$ & 36564 & (42.4\%) & $1.07(1.02-1.12)$ & $1.09(1.04-1.14)$ \\
\hline Moderate drinker (2-6 units per day) & 824 & $(4.6 \%)$ & 3520 & $(4.1 \%)$ & $1.12(1.02-1.22)$ & $1.14(1.04-1.25)$ \\
\hline Excessive drinker ( $>6$ units per day) & 155 & $(0.86 \%)$ & 656 & $(0.76 \%)$ & $1.10(0.91-1.32)$ & $1.08(0.90-1.30)$ \\
\hline Missing data & 5130 & $(28.5 \%)$ & 29137 & $(33.8 \%)$ & $0.73(0.69-0.77)$ & $0.81(0.77-0.86)$ \\
\hline \multicolumn{7}{|l|}{ BMI (tertiles) } \\
\hline 1 (median 21.5; range 11-23) & 3927 & $(21.8 \%)$ & 17015 & (19.7\%) & Baseline & \\
\hline 2 (median 25.1 ; range $23-27$ ) & 3889 & $(21.6 \%)$ & 17054 & (19.8\%) & $1.00(0.95-1.05)$ & $1.00(0.95-1.05)$ \\
\hline 3 (median 29.4; range 27-76) & 3935 & $(21.9 \%)$ & 17004 & $(19.7 \%)$ & $1.00(0.97-1.08)$ & $0.99(0.94-1.04)$ \\
\hline Missing data & 6256 & $(34.7 \%)$ & 35096 & $(40.7 \%)$ & $0.69(0.66-0.72)$ & $0.76(0.72-0.79)$ \\
\hline \multicolumn{7}{|l|}{ Medical history } \\
\hline Coronary artery disease & 3790 & $(21.0 \%)$ & 15337 & $(17.8 \%)$ & $1.24(1.19-1.29)$ & $1.02(0.98-1.06)$ \\
\hline Cerebrovascular disease & 1889 & $(10.5 \%)$ & 9082 & $(10.5 \%)$ & $0.99(0.94-1.05)$ & $0.85(0.80-0.89)$ \\
\hline Peripheral vascular disease & 1028 & $(5.7 \%)$ & 3817 & $(4.4 \%)$ & $1.32(1.23-1.42)$ & $1.09(1.01-1.17)$ \\
\hline Any atherosclerosis $†$ & 5606 & (31.1\%) & 24049 & $(27.9 \%)$ & $1.18(1.14-1.22)$ & $0.95(0.92-0.99)$ \\
\hline Hyperlipidaemia & 554 & $(3.1 \%)$ & 1963 & $(2.3 \%)$ & $1.41(1.27-1.55)$ & $1.17(1.06-1.30)$ \\
\hline Heart failure & 2188 & $(12.2 \%)$ & 9804 & (11.4\%) & $1.08(1.02-1.13)$ & $0.87(0.82-0.91)$ \\
\hline Atrial fibrillation & 1319 & $(7.3 \%)$ & 5376 & $(6.2 \%)$ & $1.19(1.12-1.27)$ & $1.01(0.94-1.07)$ \\
\hline Diabetes & 2479 & $(13.8 \%)$ & 7776 & $(9.0 \%)$ & $1.67(1.58-1.75)$ & $1.36(1.30-1.43)$ \\
\hline Cataract & 5294 & $(29.4 \%)$ & 10078 & (11.7\%) & $3.50(3.35-3.64)$ & $3.13(3.00-3.26)$ \\
\hline Hypertension & 6375 & $(35.4 \%)$ & 27081 & (31.4\%) & $1.22(1.18-1.26)$ & $1.05(1.01-1.09)$ \\
\hline \multicolumn{7}{|l|}{ Prior exposure to: } \\
\hline Any cardiovascular drug $\ddagger$ & 11209 & (62.2\%) & 49459 & $(57.4 \%)$ & $1.24(1.20-1.28)$ & $0.91(0.88-0.94)$ \\
\hline Aspirin & 4277 & $(23.8 \%)$ & 17729 & $(20.6 \%)$ & $1.22(1.17-1.27)$ & $1.0(0.96-1.04)$ \\
\hline Hormone replacement therapy & 570 & $(3.2 \%)$ & 2403 & $(2.8 \%)$ & $1.21(1.09-1.35)$ & $0.97(0.87-1.08)$ \\
\hline Fibrates & 181 & $(1.0 \%)$ & 553 & $(0.6 \%)$ & $1.59(1.34-1.89)$ & $1.30(1.09-1.55)$ \\
\hline
\end{tabular}


Table 2 Association between exposure to statins and AMD: adjusted models and dose-response relation

\begin{tabular}{|c|c|c|c|c|c|c|c|c|}
\hline \multirow[b]{2}{*}{ Statins } & \multicolumn{2}{|c|}{ Cases $(n=18007)$} & \multicolumn{2}{|c|}{$\begin{array}{l}\text { Controls } \\
\text { ( } n=86169 \text { ) }\end{array}$} & \multirow[t]{2}{*}{$\begin{array}{l}\text { Univariate odds } \\
\text { ratio }(95 \% \mathrm{Cl})\end{array}$} & \multirow[t]{2}{*}{$\begin{array}{l}\text { Odds ratio adjusted } \\
\text { for consultation } \\
\text { rate }(95 \% \mathrm{Cl})\end{array}$} & \multirow[t]{2}{*}{$\begin{array}{l}\text { Final adjusted odds } \\
\text { ratio }(95 \% \mathrm{Cl})^{*}\end{array}$} & \multirow[t]{2}{*}{ p Value } \\
\hline & & & & & & & & \\
\hline Never & 17632 & (97.9\%) & 84751 & (98.4\%) & & & & \\
\hline Ever & 375 & $(2.1 \%)$ & 1418 & $(1.6 \%)$ & $1.32(1.17-1.48)$ & $1.05(0.93-1.19)$ & $0.93(0.81-1.07)$ & 0.33 \\
\hline Within 6 months of index date & 323 & $(1.8 \%)$ & 1212 & $(1.4 \%)$ & $1.32(1.16-1.50)$ & $1.06(0.93-1.21)$ & $0.95(0.82-1.10)$ & 0.50 \\
\hline \multicolumn{9}{|l|}{ Number of prescriptions (tertiles) } \\
\hline 0 & 17632 & (97.9\%) & 84751 & (98.4\%) & Baseline & & & $0.27 \dagger$ \\
\hline $1-6$ & 125 & $(0.7 \%)$ & 490 & $(0.6 \%)$ & $1.26(1.04-1.54)$ & $1.02(0.84-1.25)$ & $0.91(0.74-1.12)$ & \\
\hline $7-19$ & 141 & $(0.8 \%)$ & 478 & $(0.6 \%)$ & $1.47(1.21-1.78)$ & $1.18(0.97-1.43)$ & $1.05(0.85-1.29)$ & \\
\hline $20+$ & 109 & $(0.6 \%)$ & 450 & $(0.5 \%)$ & $1.20(0.97-1.49)$ & $0.96(0.77-1.19)$ & $0.83(0.66-1.05)$ & \\
\hline \multicolumn{9}{|l|}{ Daily dose } \\
\hline Never & 17632 & (97.9\%) & 84751 & (98.4\%) & Baseline & & & $0.37 \dagger$ \\
\hline $10 \mathrm{mg}$ & 265 & $(1.5 \%)$ & 993 & $(1.2 \%)$ & $1.33(1.15-1.53)$ & $1.07(0.93-1.23)$ & $0.94(0.80-1.10)$ & \\
\hline $20 \mathrm{mg}$ & 95 & $(0.5 \%)$ & 370 & $(0.4 \%)$ & $1.27(1.01-1.60)$ & $1.01(0.80-1.27)$ & $0.90(0.70-1.14)$ & \\
\hline $30-40 \mathrm{mg}$ & 15 & $(0.1 \%)$ & 55 & $(0.1 \%)$ & $1.39(0.78-2.47)$ & $1.11(0.62-1.99)$ & $1.05(0.58-1.89)$ & \\
\hline
\end{tabular}

Data on potential confounding factors were derived as follows. The mean annual consultation rate for each participant was calculated, defined as the total number of consultations divided by the number of years of observation, and was included in the analyses in quintiles. Smoking was categorised as non-smoker, ex-smoker, or current smoker. Alcohol intake was categorised as non-drinker, ex-drinker, current drinker (amount unknown), rare drinker ( $<2$ units/ day), moderate drinker (2-6 units/day), excessive drinker ( $>6$ units/day). Body mass index was derived using height data and weight data measured nearest the index date. Diagnosed atherosclerotic disease was identified as coronary artery disease, cerebrovascular disease, peripheral vascular disease, and other atherosclerotic disease such as renal artery disease or where the site of the atherosclerosis was not clear. The following diagnoses were also identified: diabetes (including both type 1 and type 2), hyperlipidaemia, heart failure, and atrial fibrillation. Exposure to the following drugs was also assessed: aspirin, fibrates, and hormone replacement therapy (either oestrogen only or opposed). In addition, in order to identify as completely as possible people with cardiovascular disease, prescriptions for any cardiovascular drug (except statins and aspirin) were identified and people categorised on the basis of having received a prescription for any of these drugs prior to the index date.

Following the initial descriptive analysis, exposure to statins was modelled as a binary ever/never exposure using conditional logistic regression. We then fitted a series of bivariate models, retaining variables that were significantly associated with both AMD and with statin exposure. We adjusted for consultation rate to control for potential confounding that could occur because ascertainment of diagnosis of AMD and chance of being prescribed a statin are both influenced by frequency of consultation. Patients with missing data for covariates were treated as separate strata. The primary analysis determined the relation between the use of statins and the incidence of diagnosed AMD. We then assessed the effects of different daily doses of and total number of prescriptions for statins, and whether the effects differed by type of statin. With an estimated prevalence of exposure to statins of $1.5 \%$ and including 18000 cases we had over $90 \%$ power to detect an odds ratio of 0.8 or less at the $5 \%$ significance level.

\section{Case validation}

Some of the general practices contributing data to the GPRD provide anonymised copies of hospital letters and specialist reports on individual patients. Hospital letters were requested for a sample of 50 cases with a diagnosis of AMD recorded in their electronic GPRD record. These were reviewed by a primary care physician (LS) and a $20 \%$ sample reviewed by an ophthalmologist (UC).

\section{RESULTS}

A total of 18007 people with a diagnosis of AMD and 86169 controls were included. Descriptive details of cases and controls and univariate analyses are shown in table 1. The mean age of participants was 77.4 years and 66\% were female. The median observation period prior to the index date was 4.0 years. Cases consulted their general practitioners considerably more often than controls, reflected in the higher median annual consultation rate.

\section{Case validation}

Of the sample of 50 cases with a diagnosis of AMD recorded in their electronic GPRD record for whom we requested hospital letters, letters could not be obtained for four cases. Of the remaining 46 cases, the diagnosis of AMD was confirmed from letters or reports from eye specialists in 43 $(93.5 \%)$. Of the remaining three cases, in two a specialist diagnosis of cellophane maculopathy had been wrongly coded as AMD in the electronic primary care record, and in one case there was no further clinical evidence to support a diagnosis of AMD other than the diagnostic code in the electronic record.

\section{Association with statins}

Table 2 shows the association between exposure to statins and a diagnosis of AMD. Among cases, 2.1\% (375/18 007) had one or more recorded prescriptions for a statin, the corresponding figure for controls being 1.6\% (1418/86 169). Among those exposed, the median period of exposure to statins prior to the index date was 1.2 years for cases and 1.3 years for controls. Overall, of the 1793 participants exposed to statins, $147(8 \%)$ were exposed for more than 5 years. The crude odds ratio for the association between statin exposure and a diagnosis of AMD was 1.32 (95\% CI 1.17 to 1.48 ). The association was reduced when adjusted for mean annual consultation rate to 1.05 (95\% CI 0.93 to 1.19 ). In the final model, the odds ratio for the association between statin exposure and a diagnosis of AMD was 0.93 (95\% CI 0.81 to $1.07, \mathrm{p}=0.33$ ), adjusted for consultation rate, smoking, alcohol intake, body mass index, atherosclerotic disease, hyperlipidaemia, heart failure, diabetes mellitus, 
Table 3 Individual statins: recorded exposure and association with AMD

\begin{tabular}{lccll}
\hline Variable & Cases $(\mathbf{n = 1 8 0 0 7 )}$ & $\begin{array}{l}\text { Controls } \\
(\mathbf{n = 8 6 1 6 9 )}\end{array}$ & $\begin{array}{l}\text { Adjusted odds ratio* } \\
(\mathbf{9 5 \%} \mathbf{C I})\end{array}$ & p Value \\
\hline No statin & $17632(97.9 \%)$ & $84751(98.4 \%)$ & Baseline & \\
Any statin exposure & $375(2.1 \%)$ & $1418(1.6 \%)$ & $0.93(0.81-1.07)$ & 0.33 \\
Atorvastatin only & $29(0.16 \%)$ & $138(0.16 \%)$ & $0.76(0.50-1.15)$ & 0.19 \\
Cerivastatin only & $10(0.06 \%)$ & $53(0.06 \%)$ & $0.65(0.33-1.28)$ & 0.21 \\
Fluvastatin only & $11(0.06 \%)$ & $35(0.04 \%)$ & $1.10(0.54-2.21)$ & 0.80 \\
Pravastatin only & $47(0.26 \%)$ & $165(0.19 \%)$ & $0.99(0.70-1.39)$ & 0.95 \\
Simvastatin only & $226(1.26 \%)$ & $836(0.97 \%)$ & $0.97(0.82-1.15)$ & 0.70 \\
Mixedt & $52(0.29 \%)$ & $191(0.22 \%)$ & $0.91(0.65-1.26)$ & 0.57 \\
\hline
\end{tabular}

*Adjusted for all potential confounders significantly associated with both AMD and statin use (that is, consultation rate (quintiles), smoking, alcohol intake, BMI, atherosclerosis, hyperlipidaemia, heart failure, diabetes,

hypertension, cardiovascular drug use (excluding aspirin or statin), fibrate use). People with missing data are

included as separate strata.

†People who received a prescription for more than one type of statin.

hypertension, use of other cardiovascular drugs, and use of fibrates. The odds ratio for current use (within the past 6 months) was very similar to the odds ratio for ever use of a statin. There was no evidence that the association differed by dose or number prescriptions.

The numbers of people exposed to individual statins and the associations with AMD are shown in table 3. Of study participants with one or more prescriptions recorded for a statin, about $60 \%$ of exposed cases and 59\% of exposed controls were exposed to simvastatin only. The numbers of people receiving prescriptions for other types of statin were low, as were the numbers of participants with prescriptions recorded for more than one type of statin. None of the adjusted odds ratios approached significance and no clear pattern for different statins was evident.

\section{DISCUSSION}

The results of the present study suggest that at the usual doses used in clinical practice, short to medium term exposure to statins is not associated with a decreased risk of AMD. Although the median observation period of participants was 4 years, the duration of period of exposure to statins prior to the index date was relatively short, with only small numbers of participants exposed for more than 5 years.

The main strengths of the study were as follows. It was very large and statistically powerful compared with previous studies. Drug prescriptions from practices participating in the GPRD are generated by the practice computers ensuring the accuracy of the electronic prescribing records. Prescription data were highly detailed and recorded prior to the subject becoming a case so there was no potential for recall bias. Our validation of a random sample of cases demonstrated the high validity of a recorded diagnosis of AMD. We were able to adjust for a wide range of potential confounding factors, but these had little effect on the odds ratios obtained suggesting that there was less confounding than might have been expected. In our study, smoking was associated with an increased risk of $\mathrm{AMD}$, although the odds ratio observed was smaller than those observed in most previous studies, ${ }^{21}{ }^{22}$ suggesting we may have underestimated the effect of smoking and thus failed to fully adjust for potential confounding by smoking. We found no clear association between cardiovascular disease and AMD. The validity of a recorded diagnosis of myocardial infarction in the GPRD has been validated in a sample of over 400 cases and found to be high, ${ }^{23}$ suggesting that we would have been able to adequately adjust for any confounding effects of cardiovascular disease.

Cases included in the study were people with a clinical diagnosis of AMD and thus an important weakness is that we were not able to reliably distinguish the stage of disease nor distinguish CNV from geographic atrophy. However, we believe that we would have had a representative mix of different types of AMD as they occur in the population. Some people with AMD not yet diagnosed would not have been identified as cases and could have been included in the control group. However, of the total number of controls, the proportion with AMD would have been relatively small. There is also no reason to suspect this misclassification of controls would be differential with regards to exposure status and thus although there may have been a small reduction in power, this misclassification is unlikely to have biased the effect estimate. Clinical presentation will be determined largely by two factors. Firstly by the severity of the disease process, meaning that diagnosis is one way of assessing the stage of the disease. AMD is not an all or nothing disease: there is a continuous spectrum of severity from blinding AMD to early disease that would only be found on detailed examination. The second factor likely to determine clinical diagnosis is frequency of clinic attendance. We were able to control for the confounding effect of consultation rate on the association between statins and diagnosed AMD. We lacked information about drug exposures prior to participants registering with the GPRD. In addition our estimate of exposure was based on drugs prescribed rather than drugs known to have been taken. Although this may have led to a small degree of misclassification of exposure status, there is no reason to suspect this would be differential with regards to diagnosis and thus although there may have been a small reduction in power, any such misclassification is unlikely to have biased the effect estimate.

Assessing the evidence from the literature is beset with variations in nomenclature of AMD and of early degenerative changes such as drusen. Since the two initial small studies showing a strongly protective effect of statins against AMD, ${ }^{6}$ and of cholesterol lowering drugs against drusen progression, ${ }^{7}$ there have been a number of further studies. A cross sectional survey from Australia found that in adjusted analyses, cholesterol lowering medication (of any sort) was associated with an increased risk of age related maculopathy (defined as the presence of soft or reticular drusen or of retinal pigmentary abnormalities, with no AMD lesions), odds ratio 1.72 (95\% CI 1.18 to 2.49 ). For AMD only, a protective effect was found, but was based on 30 cases only and hence had a wide confidence interval, odds ratio 0.67 (95\% CI 0.08 to 5.40). ${ }^{8}$ The effect of different types of cholesterol lowering medication were not presented. A case control study that included 550 incident cases of people with a clinical diagnosis of AMD and 5500 matched controls with no diagnosed AMD undertaken in the USA found a markedly protective effect of statins, odds ratio 0.30 (95\% CI 0.21 to $0.45) .{ }^{9}$ Only 37 of the 550 cases had ever used a statin. A cohort study within the longitudinal Rotterdam Eye Study 
found an adjusted hazard ratio for incident age related maculopathy with statin use for greater than one year of 1.1 (95\% CI 0.7 to 1.9). ${ }^{10}$ The case definition was broad, based on any of soft distinct drusen with retinal pigmentary abnormalities, indistinct drusen, or atrophic or neovascular AMD. However, of the 419 incident cases, only 25 had any recorded statin use limiting the power of the study to detect an association. In an eye clinic based case series from San Francisco of 326 people with AMD, previously recorded statin usage was compared among people with different types of AMD ${ }^{11}$ People with CNV were significantly less likely to have previous statin use recorded than people with dry AMD or geographic atrophy, risk ratio 0.51 ( $95 \%$ CI 0.31 to 0.86 ). This suggests that statins may have a protective effect against CNV but not against other forms of AMD. In the Beaver Dam cohort study undertaken in the USA, the odds ratio for incident early age related maculopathy (defined as either soft indistinct drusen or any drusen with retinal pigmentary abnormalities) was 1.12 (95\% CI 0.47 to 2.67), while that for incident AMD (atrophic or neovascular) was 0.41 (95\% CI 0.12 to 1.45$).{ }^{12}$ For the subgroup of people who initiated statin therapy between the baseline examination in 1993-95 and the follow up examination in 1998-2000, there was a strong protective effect against late AMD, odds ratio 0.29 ( $95 \%$ CI 0.09 to 0.95 ). The relatively low power of the study is reflected in the confidence intervals of the effect estimates, and the authors concluded that further more powerful studies were needed.

We have reported the largest and most powerful study to address the relation between statin use and AMD yet undertaken. In the short and medium term statins do not seem to be associated with a decreased risk of AMD. Whether long term treatment with statins reduces the risk of AMD and whether subgroups of patients with specific forms of AMD (particularly CNV) benefit from statin therapy remains a possibility.

\section{ACKNOWLEDGEMENTS}

We would like to thank Chris Smith for help with data processing.

\footnotetext{
Authors' affiliations

L Smeeth, C Cook, A E Fletcher, Department of Epidemiology and Population Health, London School of Hygiene and Tropical Medicine, London, United Kingdom

U Chakravarthy, Centre for Vision Science, The Queen's University of Belfast. Institute of Clinical Science, Royal Hospitals, Belfast, UK

R Hubbard, Division of Respiratory Medicine, University of Nottingham, Nottingham City Hospital, UK

Funding: the study was funded by the United Kingdom Medical Research Council (MRC). Liam Smeeth is supported by a Medical Research Council
}

clinician scientist fellowship. Richard Hubbard is supported by a Wellcome Trust advanced fellowship.

Competing interests: none declared

\section{REFERENCES}

1 Congdon NG, Friedman DS, Lietman T. Important causes of visual impairment in the world today. JAMA 2003;290:2057-60.

2 Age-Related Eye Disease Study Research Group. A randomized, placebocontrolled, clinical trial of high-dose supplementation with vitamins $C$ and $E$, beta carotene, and zinc for age-related macular degeneration and vision loss: AREDS report no 8. Arch Ophthalmol 2001;119:1417-36.

3 Chopdar A, Chakravarthy U, Verma D. Age related macular degeneration. BMJ 2003;326:485-8

4 Delcourt C, Michel F, Colvez A, et al. Associations of cardiovascular disease and its risk factors with age-related macular degeneration: the POLA study. Ophthalmic Epidemiol 2001;8:237-49.

5 Snow KK, Seddon JM. Do age-related macular degeneration and cardiovascular disease share common antecedents? Ophthalmic Epidemiol 1999;6:125-43.

6 Hall NF, Gale CR, Syddall H, et al. Risk of macular degeneration in users of statins: cross sectional study. BMJ 2001;323:375-6.

7 McCarty CA, Mukesh BN, Guymer RH, et al. Cholesterol-lowering medications reduce the risk of age-related maculopathy progression. Med J Aust $2001 ; 175: 340$.

8 McCarty CA, Mukesh BN, Fu CL, et al. Risk factors for age-related maculopathy: the Visual Impairment Project. Arch Ophthalmol 2001;119:1455-62

9 McGwin G Jr, Owsley C, Curcio CA, et al. The association between statin use and age related maculopathy. Br J Ophthalmol 2003;87:1121-5.

10 van Leeuwen R, Vingerling JR, Hofman A, et al. Cholesterol lowering drugs and risk of age related maculopathy: prospective cohort study with cumulative exposure measurement. BMJ 2003;326:255-6.

11 Wilson HL, Schwartz DM, Bhatt HR, et al. Statin and aspirin therapy are associated with decreased rates of choroidal neovascularization among patients with age-related macular degeneration. Am J Ophthalmol 2004;137:615-24.

12 Klein R, Klein BE, Tomany SC, et al. Relation of statin use to the 5 -year incidence and progression of age-related maculopathy. Arch Ophthalmol 2003;121:1151-5.

13 McGwin G Jr, Xie A, Owsley C. The use of cholesterol-lowering medications and age-related macular degeneration. Ophthalmology 2005;1 12:488-94.

14 van Leeuwen R, Vingerling JR, de Jong PT. Risk of macular degeneration with statin use should be interpreted with caution. BMJ 2001;323:1308.

15 Walley T, Mantgani A. The UK General Practice Research Database. Lancet 1997;350:1097-9.

16 Office for National Statistics. Key health statistics from general practice 1996 (Series MB6 No 1). London: Office for National Statistics, 1998.

17 Jick H, Jick SS, Derby LE. Validation of information recorded on general practitioner based computerised data resource in the United Kingdom. BMJ $1991 ; 302: 766-8$

18 Nazareth I, King M, Haines A, et al. Accuracy of diagnosis of psychosis on a general practice computer system. BMJ 1993;307:32-4.

19 Kaye JA, Derby LE, del MM-M, et al. The incidence of breast cancer in the General Practice Research Database compared with national cancer registration data. $\mathrm{Br} J$ Cancer 2000;83:1556-8.

20 Jick SS, Kaye JA, Vasilakis-Scaramozza C, et al. Validity of the general practice research database. Pharmacotherapy 2003;23:686-9.

21 Klein $\mathbf{R}$, Klein BE, Linton KL, et al. The Beaver Dam Eye Study: the relation of age-related maculopathy to smoking. Am J Epidemiol 1993;137:190-200.

22 Tomany SC, Wang JJ, van Leeuwen R, et al. Risk factors for incident agerelated macular degeneration: pooled findings from 3 continents. Ophthalmology 2004;111:1280-7.

23 Meier CR, Jick SS, Derby LE, et al. Acute respiratory-tract infections and risk of first-time acute myocardial infarction. Lancet 1998;351:1467-71. 